Ytrebø, L.M. et al., 2004. Contractile response of femoral arteries in pigs with acute liver failure. Scandinavian journal of gastroenterology, 39(10), p.1000-1004.

\title{
CONTRACTILE RESPONSE OF FEMORAL ARTERIES IN PIGS WITH ACUTE LIVER FAILURE
}

\author{
L. M. Ytrebø, S. Ekse, S. Sen, C. Rose, G. I. Nedredal, O.-M. Fuskevåg, R. Jalan and A. \\ Revhaug
}

${ }^{1}$ Depts. of Digestive Surgery and Clinical Pharmacology, University Hospital Northern Norway, Tromsø, Norway ${ }^{2}$ Liver Failure Group, Institute of Hepatology, University College London, London, UK ${ }^{3}$ Dept. of Cellular Neuroscience, Max-Delbrück Centre for Molecular Medicine, Berlin, Germany Correspondence: Lars Marius, Ytrebø, M.D., Ph.D, Liver Failure Group, Institute of Hepatology, University College London, 69-75 Chenies Mews, London, WC1E 6HX, UK, +44 (0)207 3800405 larsmy@fagmed.uit.no

\section{ABSTRACT}

Background: Acute liver failure (ALF) is characterized haemodynamically by a progressive hyperdynamic circulation. The pathophysiological mechanism is unknown, but impaired contractility of vascular smooth muscle may play an important role. The aim of this study was to evaluate the vascular response to stimulation with norepinephrine and angiotensin II in endothelium-denuded femoral artery rings. Methods: Norwegian Landrace pigs weighing $27.1 \pm 0.5 \mathrm{~kg}$ (mean $\pm s_{x}$ (standard error of the mean)) were used. ALF was induced by performing a portacaval shunt followed by ligation of the hepatic arteries $(n=6)$. Sham-operated animals served as controls $(n=$ 5). Cumulative isometric concentration contraction curves were obtained after in vitro stimulation of the femoral artery rings with either angiotensin II $\left(10^{-13}-10^{-5} \mathrm{~mol} / \mathrm{L}\right)$ or norepinephrine $\left(10^{-13}-10^{-3} \mathrm{~mol} / \mathrm{L}\right)$. Results: Pigs suffering from ALF developed a hyperdynamic circulation with an increased cardiac index $(P=0.017)$ and decreased systemic vascular resistance index $(P=0.015)$. Studies of the hind leg revealed a decreased vascular resistance index and increased blood flow compared to sham-operated controls $(P=0.003$ and $P=0.01$, respectively). Angiotensin II caused a concentration-dependent contraction of the arterial segments, with no significant differences in vascular responses between the two groups. Maximum force generated did not differ ( $55 \pm 7$ versus $56 \pm 7 \mathrm{mN}, P$ $=0.95)$. Furthermore, there were no differences for norepinephrine in the cumulative concentration-response curves and the maximum contractile force was not significantly different ( $87 \pm 8$ versus $93 \pm 16 \mathrm{mN}, P=0.55$ ). Conclusions: This study documents for the first time that there are no signs of endothelium-independent peripheral vascular hyporesponsiveness to angiotensin II and norepinephrine in pigs with ALF.

Keywords Angiotensin II, haemodynamic, norepinephrine, vascular physiology

\section{Introduction}

Patients suffering from acute liver failure (ALF) develop a hyperdynamic circulation (1). Although the occurrence of arterial hypotension is widely recognized (2), the exact pathogenesis has still to be defined. Compensatory activation of neurohumoral reflexes involving the sympathetic nervous system and renin-angiotensin axis normally counteract for the loss of vascular tone (3). However, despite increased catecholamine levels in plasma, patients remain hypotensive with high cardiac output and low systemic vascular resistance (4). Interventions to increase blood pressure are often necessary and catecholamines (e.g. epinephrine, norepinephrine and dopamine) are frequently given intravenously $(5,6)$.

We formerly established a large animal (porcine) model of ALF, induced by hepatic devascularization, which is characterized by a sharp increase in serum bilirubin and amino-transferases, coagulopathy, intracranial hypertension and a hyperdynamic circulation (7). In this model we found a significant increased blood flow to the hind leg and a decreased hind leg vascular resistance as the hyperdynamic circulation progressed, which is not

The final publication is available at http://dx.doi.org/10.1080/00365520410003254 
Ytrebø, L.M. et al., 2004. Contractile response of femoral arteries in pigs with acute liver failure. Scandinavian journal of gastroenterology, 39(10), p.1000-1004.

considered as an adequate physiologic response to the decline in mean arterial blood pressure (MAP). To date, neither animal nor human studies have explored the pathophysiology behind this phenomenon. Two human studies have demonstrated increased circulating levels of nitric oxide (NO) in ALF $(8,9)$. Furthermore, nitric oxide synthase (NOS) seems to be upregulated in the hepatic arteries of ALF patients (10). Accordingly, excessive release of NO appears to be an important endothelium-dependent pathogenetic factor for the haemodynamic derangement observed. However, impaired contractility of vascular smooth muscle tone (endothelium independent) might play a significant role as well, though there are no studies that specifically have addressed this issue. Therefore, we decided to use our porcine model to investigate the peripheral vascular response to important vasoconstrictors in ALF.

We hypothesized that arterial hypotension in ALF might be caused partly by a vascular hyporesponsiveness to vasoconstrictors. Thus the aim of this study was to investigate the in vitro vascular response to angiotensin II and norepinephrine in denuded femoral artery rings (thus excluding interference from endothelium-derived vasoactive factors) in a porcine model of ALF (7) and in sham-operated controls.

\section{METHODS}

The study was performed with the approval of the Norwegian Experimental Animal Board. Eleven Norwegian female Landrace pigs, weighing $27.1 \pm 0.5 \mathrm{~kg}$ (mean $\pm \mathrm{sx}$ (standard error of the mean)) were used. Details regarding the animal room and preparation of the animals have been published elsewhere (11). The animals were randomized into ALF or sham surgery groups by drawing lots before the laparatomy. ALF was induced by performing a portacaval shunt followed by ligation of the hepatic arteries $(n=6)$. Sham-operated animals served as controls $(n=5)$. The surgical procedure has been described elsewhere (12). Briefly, MAP and heart rate were measured by introducing a Portex polyethylene tubing (ID $0.58 \mathrm{~mm}$, OD $0.96 \mathrm{~mm}$, Portex Ltd., Hythe, Kent, UK) into the abdominal aorta. A 5 French Edwards Swan-Ganz catheter (Baxter Healthcare Corp, Irvine, Calif., USA) was floated into the pulmonary artery via the right external jugular vein to measure cardiac index (CI) using the thermodilution technique. A perivascular, ultrasonic, transit time flow probe (CardioMed Systems, Medistim A/S, Oslo, Norway) was placed around the right iliac artery for blood flow measure-ments. Systemic vascular resistance index (SVRI) was calculated as: (MAP-CVP)/CI. Hind-leg resistance was calculated as (MAP-CVP)/iliac artery flow.

\section{Preparation of the vessels}

The experiments were terminated after $6 \mathrm{~h}$, and an incision was made medially on the right leg where the right femoral artery was dissected free. The artery was clamped and immediately transferred into oxygenized (95\% 02, 5\% CO2) modified ice-cold Krebs-Henseleit solution (in mmol/L: $\mathrm{NaCl} 118.3, \mathrm{KCl} 4.7, \mathrm{CaCl} 22.5, \mathrm{MgSO} 41.17$, KH2P021.18, NaHC0325.0, EDTA 0.026, glucose 11.1). The vessels were cleaned of connective tissue under a magnifying glass and the endothelium was removed mechanically by gently rubbing with a rough steel rod. The vessels were cut into 2-mm-wide rings and mounted in an organ bath filled with modified Krebs-Henseleit solution, which was continuously exchanged with oxygenized (95\% 02, 5\% CO2) modified Krebs-Henseleit buffer via a syringe pump. Temperature was maintained at $37^{\circ} \mathrm{C}$ by using an outer water jacket. The vasoconstriction was measured with an isometric force transducer (Fort 10; World Precision Instruments, Berlin, Germany). Transducer outputs were amplified (Gould Inc., Recording System, Cleveland, Ohio, USA) and recorded electronically using a LabVIEW program that was developed in-house (Danielsen, Arild, arildd@fagmed.uit.no). During an equilibration period of $1 \mathrm{~h}$, a baseline tension of $20 \mathrm{mN}$ was adjusted. Acetylcholine $\left(10^{-5} \mathrm{~mol} / \mathrm{L}\right)$ was added to prove the absence of intact endothelium. In order to ensure viability of the vessels, $40 \mathrm{mmol} / \mathrm{L} \mathrm{KCl}$ was perfused through the organ bath at the end of the experimental period.

\section{Vascular response to stimulation with angiotensin II and norepinephrine}

The contractile response to angiotensin II and norepinephrine was assessed in the denuded femoral artery rings obtained from 11 pigs (6 ALF, 5 sham). The drugs were dissolved in oxygenized modified Krebs-Henseleit buffer and added via a syringe pump to the organ bath at a constant infusion rate of $120 \mathrm{~mL} \mathrm{~h}^{-1}$.

The final publication is available at http://dx.doi.org/10.1080/00365520410003254 
Ytrebø, L.M. et al., 2004. Contractile response of femoral arteries in pigs with acute liver failure. Scandinavian journal of gastroenterology, 39(10), p.1000-1004.

Cumulative concentration-response curves for angiotensin II ( $10^{-13}$ to $\left.10^{-5} \mathrm{~mol} / \mathrm{L}\right)$ and norepinephrine $\left(10^{-13}\right.$ to $10^{-3}$ $\mathrm{mol} / \mathrm{L}$ ) were plotted. The concentration ranges were identified as appropriate from previous pilot experiments and in accordance with concentrations applied to human hepatic arteries (13). Contraction force was monitored continuously and maximum force was noted within $10 \mathrm{~min}$. These readings were used to plot a cumulative doseresponse curve. No force adjustments were made between the different concentrations. Norepinephrine and angiotensin II were purchased from Sigma-Aldrich (Oslo, Norway).

\section{Determination of urinary norepinephrine}

Analysis of norepinephrine in urine was performed as previously described by Kushnir et al. (14). Urinary norepinephrine excretion was calculated as (norepinephrine) urine output.

\section{Statistical analysis}

Data are presented as the mean \pm sx. Vasoconstriction is expressed in milli Newton $(\mathrm{mN})$. The unpaired Student's $\mathrm{t}$ test was applied to test for differences in maximum force generated by the vessels. Two-way (group and time) analysis of variance for repeated measures was applied for other haemodynamic and biochemical variables. PG denotes the effect of group and PGT denotes the interaction for group and time. The unpaired Student's t test was applied to test for differences in haemodynamic and biochemical variables at $t=6 \mathrm{~h}$. A P value 0.05 was taken to determine statistical significance. Statistical analyses were performed with the SPSS 11.0 software package (SPSS, Chicago, Ill.) and Excel 5.0 (Microsoft).

\section{RESULTS}

\section{Cardiovascular changes}

The cardiovascular changes are summarized in Table I. Pigs with ALF developed evidence of a hyperdynamic circulation with increasing CI and decreasing SVRI. Furthermore, there was a significant difference in iliac artery flow between the groups, and a highly significant decrease in hindleg resistance compared with that in shamoperated controls. Despite a slightly decreased MAP in the sham group during the experimental period, MAP was significantly lower in the ALF group $\mathrm{t}=6 \mathrm{~h}$.

\section{Biochemical changes}

Pigs with ALF developed hyperbilirubinaemia. Creatinine levels increased in the ALF group, which caused a significant group effect (Table II). There was no significant interaction for norepinephrine excretion in urine, but the ALF group tended to have higher norepinephrine levels over the time period studied $(\mathrm{PG}=0.056)$. However, norepinephrine excretion rate was significantly higher in the ALF group at $t=6 \mathrm{~h}(\mathrm{P}=0.035)$. 
Ytrebø, L.M. et al., 2004. Contractile response of femoral arteries in pigs with acute liver failure. Scandinavian journal of gastroenterology, 39(10), p.1000-1004.

Table I. Haemodynamic characteristics of pigs with acute liver failure (ALF; $n=6)$ and sham-operated controls $(n=5 \mathrm{~h})$

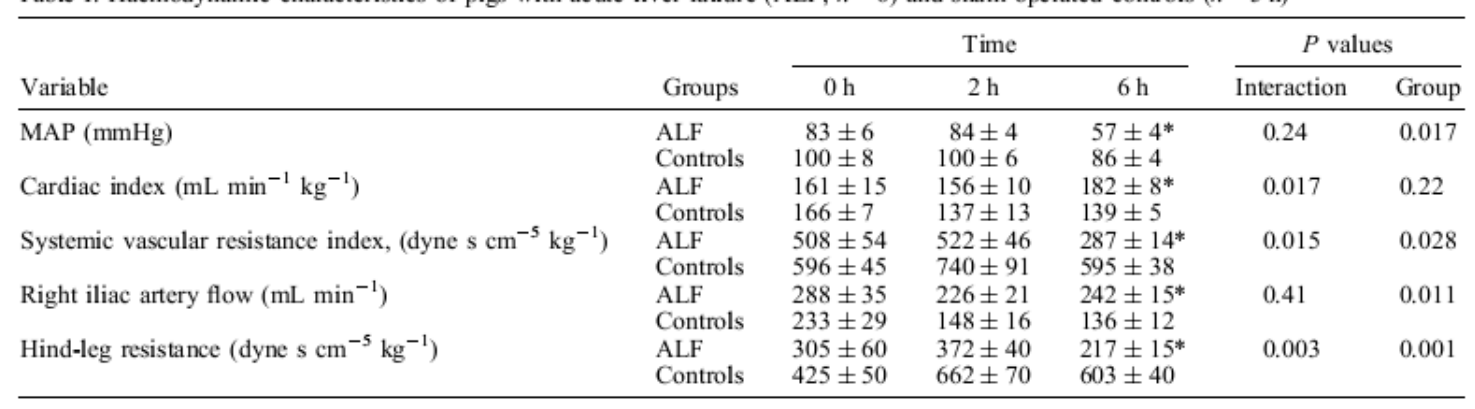

$\mathrm{MAP}=$ mean arterial pressure; $\mathrm{ALF}=$ acute liver failure; ANOVA: analysis of variance for repeated measures.

$* P<0.01$, independent $t$ test at $t=6 \mathrm{~h}$.

Table II. Biochemical characteristics of pigs with acute liver failure (ALF; $n=6)$ and sham-operated controls $(n=5 \mathrm{~h})$

\begin{tabular}{|c|c|c|c|c|c|c|}
\hline Variable & Groups & \multicolumn{3}{|c|}{ Time } & \multicolumn{2}{|c|}{$P$ values } \\
\hline Bilinubin $\left(\mu \mathrm{mol} \mathrm{L}^{-1}\right)$ & $\begin{array}{l}\text { ALF } \\
\text { Controls }\end{array}$ & $\begin{array}{l}4 \pm 1 \\
2 \pm 0.3\end{array}$ & $\begin{aligned} 11 & \pm 1 \\
3 & \pm 0.3\end{aligned}$ & $\begin{aligned} 17 & \pm 1^{*} \\
6 & \pm 0.2\end{aligned}$ & $<0.001$ & $<0.001$ \\
\hline Creatinine $\left(\mu \mathrm{mol} \mathrm{L}^{-1}\right)$ & $\begin{array}{l}\text { ALF } \\
\text { Controls }\end{array}$ & $\begin{array}{l}91 \pm 4 \\
75 \pm 3\end{array}$ & $\begin{aligned} 101 & \pm 6 \\
77 & \pm 3\end{aligned}$ & $\begin{array}{l}118 \pm 13^{*} \\
74 \pm 4\end{array}$ & 0.08 & 0.012 \\
\hline Urinary noradrenalin excretion $\left(\mu \mathrm{mol} \mathrm{h}^{-1}\right)$ & $\begin{array}{l}\text { ALF } \\
\text { Controls }\end{array}$ & $\begin{array}{c}1.2 \pm 0.4 \\
0.94 \pm 0.08\end{array}$ & $\begin{array}{r}1.5 \pm 0.2 \\
0.90 \pm 0.3\end{array}$ & $\begin{array}{r}1.6 \pm 0.3^{*} \\
0.62 \pm 0.07\end{array}$ & 0.49 & 0.056 \\
\hline
\end{tabular}

$\mathrm{ALF}=$ acute liver failure.

${ }^{*} P<0.05$, independent $t$ test at $t=6 \mathrm{~h}$.

\section{In vitro vascular response}

Acetylcholine \& $\mathrm{KCl}$. Endothelial denudation was con-firmed by adding acetylcholine to the organ bath after a stabilization period of $1 \mathrm{~h}$. No vascular relaxation was observed. Intact vascular viability was tested before termination of the experiments by adding $40 \mathrm{mmol} / \mathrm{L} \mathrm{KCl}$ to the organ bath. All vessels were viable with a significant increase in the contraction force after stimulation (data here as mean \pm sx for each group).

Angiotensin II. Stimulation with angiotensin II caused a concentration-dependent contraction of the arterial segments. There were no differences in vascular responses between the two vessel groups (Fig. 1). The contractile response to $\geq 10^{-5} \mathrm{~mol} / \mathrm{L}$ angiotensin II was less than that for $10^{-7} \mathrm{~mol} / \mathrm{L}$, which complies with a plateau phase in the contractile response. Maximum force generated after stimulation with angiotensin II did not differ between the ALF and control groups ( $55 \pm 7$ and $56 \pm 7 \mathrm{mN}$, respectively, $\mathrm{P}=0.95$ ) (Fig. 2). A plateau effect was observed at concentrations $105 \mathrm{~mol} / \mathrm{L}$.

Norepinephrine. There was no significant difference between the groups for norepinephrine $103 \mathrm{~mol} / \mathrm{L}$. Maxi-mum force generated after stimulation with norepinephrine did not differ between the two vessel groups (87 \pm 8 and 93 $\pm 16 \mathrm{mN}$, respectively, $\mathrm{P}=0.55$ ).

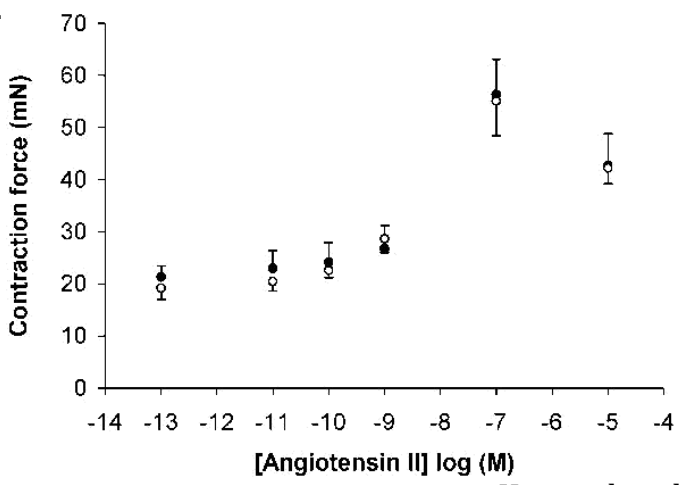

Fig. 1. Cumulative concentration-response curves to angiotensin II in isolated, endothelium-denuded femoral artery

The final publication is available at http://dx.doi.org/10.1080/00365520410003254 
Ytrebø, L.M. et al., 2004. Contractile response of femoral arteries in pigs with acute liver failure. Scandinavian journal of gastroenterology, 39(10), p.1000-1004.

rings of pigs with acute liver failure (ALF) and sham-operated controls. Contraction forces are expressed in milli Newton as the mean \pm sx (standard error of the mean). Controls; ALF.

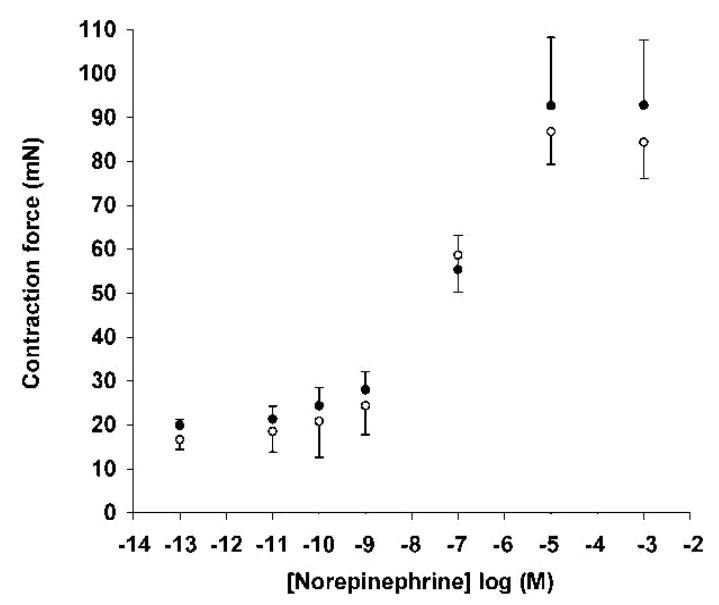

Fig. 2. Cumulative concentration-response curves to norepinephrine in isolated, endothelium-denuded femoral artery rings of pigs with acute liver failure (ALF) and sham-operated controls. Contraction forces are expressed in milli Newton as the mean $\pm \mathrm{sx}$ (standard error of the mean). Controls; ALF.

\section{DISCUSSION}

We investigated the vascular response to angiotensin II and norepinephrine in denuded femoral arteries from pigs with ALF. Our results show that neither the maximal contraction force nor the dose-response curves differed between pigs with ALF and sham-operated controls. Accordingly, we found no evidence for peripheral vascular hyporesponsiveness to these vasoconstrictor neurohormones in ALF.

The arterial hyporesponsiveness observed in other studies in liver failure may result either from the presence of excessive vasorelaxing factors or an impairment of the contractile apparatus of the vascular smooth muscle cells. However, a combination of these mechanisms is suggested. Smith et al. found impaired vascular response to phenylephrine in hepatic arteries from ALF patients (10), but this was not confirmed in a recently published human study of omental arteries (15). Since both phenylephrine and norepinephrine act via the 1-receptors, we assume that our results are comparable and a difference (if any) would have been detectable over the concentration and time range investigated. Smith et al. performed their study on intact hepatic artery rings, but they were unable to demonstrate any relaxation to stimulation with acetylcholine, which implies a lack of endothelium. Therefore, the differences in the results from our study and those of Smith et al. are likely to reflect differences in the responsiveness of vessels obtained from different vascular beds.

Peripheral vascular response to angiotensin II has not previously been described either in experimental nor in human ALF, but Schepke et al. demonstrated vascular hypo-responsiveness to angiotensin II in hepatic arteries in humans with cirrhosis (13). This finding has recently been confirmed in a study of patients with cirrhosis, where hyporesponsiveness to angiotensin II was demonstrated in the forearm, but this hyporesponsiveness was corrected following inhibition of NOS, suggesting that this hyporesponsiveness is principally due to enhanced NO generation (16).

Our porcine ALF model exhibited a hyperdynamic circulation with increasing cardiac output and decreasing systemic vascular resistance during the first $6 \mathrm{~h}$ after ALF induction, which is a valid model for the purpose of investigating vascular function in ALF. The present data document a peripheral vascular dilatation in the ALF group compared to sham-operated controls, which is in accordance with our previously published data (7). Furthermore, these animals were rapidly deteriorating with increasing plasma bilirubin levels, along with increasing serum

The final publication is available at http://dx.doi.org/10.1080/00365520410003254 
Ytrebø, L.M. et al., 2004. Contractile response of femoral arteries in pigs with acute liver failure. Scandinavian journal of gastroenterology, 39(10), p.1000-1004.

creatinine levels. Urinary norepinephrine excretion was measured to monitor the amounts of circulating endogenous norepinephrine levels in the animals. The Data confirm our presumption that ALF pigs do have increased catecholamine levels, though still too inadequate to increase MAP.

Our data show a highly significant decrease in both systemic and regional (hind leg) vascular resistance during the 6$\mathrm{h}$ experimental period after ALF induction. Furthermore, our data suggest a normal response to important vascular smooth muscle constrictors. Accordingly, the peripheral vasodilation in our model seems not to be due to hyporesponsiveness to norepinephrine or angiotensin II. However, our results are based on in vitro experiments on the femoral arteries and it is impossible to conclude from these experiments what the vascular responses might be in other vascular beds. It remains unknown whether smaller resistance arterioles from the hind leg or vessels from other vascular beds exhibit a similar pattern upon stimulation with angiotensin and norepinephrine. These results, however, pave the way for further studies of the pathophysiological basis for the circulatory collapse in ALF. First, assessment of vascular responsiveness should be further examined in vessels obtained from different vascular beds in ALF. Secondly, the impact of endothelium-derived vasodilators such as NO and prostaglandins on vascular function need to be further investigated. NOS is up-regulated in hepatic arteries and increased levels of NO have been demonstrated in humans with ALF $(8,9)$. Moreover, prostaglandins are widely known to play an important role in the autoregulation of vasogenic tone. Inducible cyclooxygenase (COX-2) was recently demonstrated to be upregulated in omental arteries harvested from ALF patients, and selective inhibition of COX-2 had a significant influence on both norepinephrine and thromboxane A2-elicited vasoconstriction in vitro (15). However, the latter study, which was performed on splanchnic vessels, does not necessarily reflect the situation in peripheral vessels. In conclusion, this study documents for the first time that there is no evidence of peripheral vascular hyporesponsiveness of endothelium-denuded vessels to angiotensin II and norepinephrine in pigs with ALF.

\section{ACKNOWLEDGEMENTS}

We gratefully acknowledge Knut Steines and Fredrik Bergheim for their support in setting up the vascular contractility measurements. Hege Hagerup, Ellinor Hareide, Hanne Mæhre and Torfinn Solvang are acknowledged for excellent technical assistance during the experiments. We also thank the Norwegian Research Council, the Sir Siegmund Warburg Voluntary Settlement and the Liver Research Foundation for financial support.

\section{REFERENCES}

1. Ellis A, Wendon J. Circulatory, respiratory, cerebral, and renal derangements in acute liver failure: pathophysiology and management. Semin Liver Dis 1996;16:379-88.

2. Trewby PN, Williams R. Pathophysiology of hypotension in patients with fulminant hepatic failure. Gut 1977;18:1021-6.

3. Guyton AC, Hall JE. The circulation. In: Guyton AC, Hall JE, editors. Textbook of medical physiology. Philadelphia: WB Saunders; 2000. p. 161-297.

4. Makin AJ, Hughes RD, Williams R. Systemic and hepatic hemodynamic changes in acute liver injury. Am J Physiol 1997; 272:G617-25.

5. Wendon JA, Harrison PM, Keays R, Gimson AE, Alexander GJ, Williams R. Effects of vasopressor agents and epoprostenol on systemic hemodynamics and oxygen transport in fulminant hepatic failure. Hepatology 1992;15:1067-71.

6. Clemmesen JO, Galatius S, Skak C, Dalgaard P, Larsen FS, Ott P. The effect of increasing blood pressure with dopamine on systemic, splanchnic, and lower extremity hemodynamics in patients with acute liver failure. Scand J Gastroenterol 1999;34: 917-27.

7. Ytrebø LM, Nedredal GI, Langbakk B, Revhaug A. An experi-mental large animal model for the assessment of bioartificial liver support systems in fulminant hepatic failure. Scand J Gastroenterol 2002;37:1077-88.

8. Harrison P, Wendon J, Williams R. Evidence of increased guanylate cyclase activation by acetylcysteine in fulminant hepatic failure. Hepatology 1996;23:1067-72.

The final publication is available at http://dx.doi.org/10.1080/00365520410003254 
Ytrebø, L.M. et al., 2004. Contractile response of femoral arteries in pigs with acute liver failure. Scandinavian journal of gastroenterology, 39(10), p.1000-1004.

9. Schneider F, Lutun P, Boudjema K, Wolf P, Tempe JD. In vivo evidence of enhanced guanylyl cyclase activation during the hyperdynamic circulation of acute liver failure. Hepatology 1994;19:38-44.

10. Smith RE, Robinson NM, McPeake JR, Baylis SA, Charles IG, Heaton ND, et al. Induction and role of NO synthase in hypotensive hepatic failure. Arterioscler Thromb Vasc Biol 1997;17: 3079-82.

11. Ytrebø LM, Korvald C, Nedredal GI, Elvenes OP, Nielsen Grymyr OJ, Revhaug A. N-acetylcysteine increases cerebral perfusion pressure in pigs with fulminant hepatic failure. Crit Care Med 2001;29:1989-95.

12. Ytrebø LM, Ingebrigtsen T, Nedredal GI, Elvenes OP, Korvald C, Romner B, et al. Protein S-100 : a biochemical marker for increased intracranial pressure in pigs with acute hepatic failure. Scand J Gastroenterol 2000;35:546-51.

13. Schepke M, Heller J, Paschke S, Thomas J, Wolff M, Neef M, et al. Contractile hyporesponsiveness of hepatic arteries in humans with cirrhosis: evidence for a receptor-specific mechanism. Hepatology 2001;34:884-8.

14. Kushnir MM, Urry FM, Frank EL, Roberts WL, Shushan B. Analysis of catecholamines in urine by positive-ion electrospray tandem mass spectrometrometry. Clin Chem 2002;48:323-31.

15. Tabernero A, Schneider F, Potenza MA, Fidi-Soa Randriamboa-vonjy V, Chasserot S, Wolf P, et al. Cyclooxygenase-2 and inducible nitric oxide synthase in omental arteries harvested from patients with severe liver diseases: immunolocalization and influence on vascular tone. Intensive Care Med 2003;29:262-70.

16. Helmy A, Newby DE, Jalan R, Johnston NR, Hayes PC, Webb DJ. Nitric oxide mediates the reduced vasoconstrictor response to angiotensin II in patients with preascitic cirrhosis. J Hepatol 2003;38:44-50.

The final publication is available at http://dx.doi.org/10.1080/00365520410003254 